\title{
Rhinosinusitis
}

\section{Mit Pflanzenkraft gegen die Symptome}

- Rhino-, Corona-, Influenza-, RespiratorySyncytial- und Parainfluenzaviren sind die häufigsten Erreger von Entzündungen im Nasenraum. Diese Erkrankungen werden zu $90 \%$ durch Viren verursacht. Deshalb sind bei einer akuten Rhinosinusitis, selbst bei einem schweren Verlauf, Antibiotika in den meisten Fällen nicht indiziert, teilte das Unternehmen Bionorica SE mit. Laut

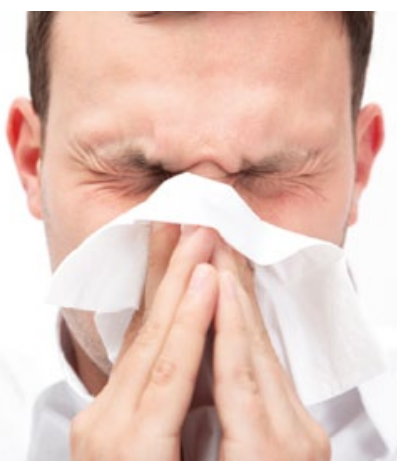

Antibiotika sind hier meist nicht indiziert. einer Erhebung in 30 deutschen Hausarztpraxen werde bei $64 \%$ der Patienten mit Sinusitis und bei $18 \%$ mit Erkältung trotzdem ein Antibiotikum verschrieben.

\section{Antibiotika nützen wenig}

Dass eine Antibiotikatherapie wenig nütze, habe eine US-amerikanische Studie ergeben. 186 Patienten mit akuter Rhinosinusitis erhielten entweder Amoxicillin oder Placebo zusätzlich zur symptomatischen Therapie. Das Ergebnis: Die Gabe des Antibiotikums linderte weder signifikant stärker die Symptome noch waren die Patienten schneller wieder gesund. Den Forschern zufolge brachte die Antibiotikatherapie den Patienten keinen Nutzen. Aufgrund des Resistenzrisikos sowie möglicher unerwünschter Wirkungen durch das Antibiotikum selbst sollte dessen Einsatz im Rahmen einer Rhinosinusitis daher möglichst vermieden werden.
Kurz notiert

Venöse Thromboembolie $>$ Die neue Service-Homepage, www.innohep. de, bietet Ärzten und Apothekern fundierte Fachinformationen rund um die Prophylaxe und Therapie venöser Thromboembolien sowie das niedermolekulare Heparin Tinzaparin (innohep ${ }^{\oplus}$ ).

Leo Pharma

Eine alternative Therapieoption bei akuter oder chronischer Rhinosinusitis sei das pflanzliche Medikament Sinupret ${ }^{\oplus}$. Es besitze ein Wirkprofil, das bestens auf die Behandlung von Patienten mit Nasennebenhöhlenentzündung abgestimmt sei: Die Wirkstoffkombination aus fünf Pflanzen wirke sekretolytisch, antiinflammatorisch, antiviral und antibakteriell - und damit sowohl gegen die Symptome als auch gegen die Ursachen der Rhinosinusitis.

Red.

Quelle: Informationen von Bionorica SE

\section{Hemmung der tubulären Glukoserückresorption \\ Neues Behandlungskonzept für Typ-2-Diabetiker}

- „Beim Typ-2-Diabetiker ist die Niere nicht nur Opfer, sondern auch Täter", sagte Prof. Hermann Haller, Hannover. Bei der Glukohomöostase spiele die Niere eine wichtige Rolle. Die renale Glukoseproduktion im Nüchternzustand macht ca. 25\% der gesamten Glukosemenge aus. Darüber hinaus resorbiert die Niere täglich insulinunabhängig $180 \mathrm{~g}$ glomerulär filtrierte Glukose.

Gesteuert werden die tubulären Transportprozesse durch Transportproteine, die Natrium-Glukose-Kotransporter (SGLT).,,Der vorwiegend im proximalen Nierentubulus lokalisierte Natrium-Glukose-Kotransporter SGLT-2 bestreitet ca. $90 \%$ der tubulären Glukoserückresorption", so Haller.

Typ-2-Diabetiker zeigen aufgrund einer erhöhten Expression von SGLT-2 eine vermehrte renale Glukoneogenese und eine gesteigerte tubuläre Rückresorption von Glukose. „Dadurch tragen diese renalen pa- thogenetischen Mechanismen wesentlich zur diabetischen Hyperglykämie bei", so Haller. Die Hemmung des SGLT-2-Transportersystems biete eine vielversprechende Behandlungsoption für Typ-2-Diabetiker.

\section{Blutzucker, $\mathrm{HbA}_{1 \mathrm{c}}$ und}

\section{Körpergewicht werden gesenkt}

Zu dieser Substanzgruppe gehört Dapagliflozin, das selektiv und potent SGLT-2 hemmt. Entsprechende Studienergebnisse bei Typ-2-Diabetikern zeigen, dass sowohl in der Monotherapie als auch in Kombination mit Metformin nicht nur die Glukosurie gesteigert, sondern auch die Blutzucker- und $\mathrm{HbA}_{1 \mathrm{c}}$-Werte gesenkt werden können bei gleichzeitiger Gewichtsabnahme. Darüber hinaus wurde eine leichte blutdrucksenkende Wirkung dokumentiert. In Kombination mit Insulin musste im weiteren Verlauf die Insulindosierung weniger stark erhöht werden als unter Place- bo. Die Substanz führt nicht zu einer Hypoglykämie.

Beobachtungen über zwei Jahre zeigen, dass die günstigen Wirkungen auf Blutzucker, $\mathrm{HbA}_{1 c}$-Wert und Gewicht dauerhaft erhalten bleiben. Bezüglich der Sicherheit ergaben sich keine Hinweise für ein erhöhtes kardiovaskuläres Risiko, auch das Interaktionspotenzial ist gering. Allerdings war unter Dapagliflozin das Risiko für eine Urogenitalinfektion erhöht.

Dapagliflozin wird einmal täglich gegeben. Seine Wirkung ist abhängig von der Nierenfunktion, d.h. bei chronischer Niereninsuffizienz (GFR $<60 \mathrm{ml} / \mathrm{Min}$.) ist die Wirksamkeit der Substanz schwächer.

- Dr. med. Peter Stiefelhagen

Quelle: Satellitensymposium „Innovative und optimierte Behandlungskonzepte des Typ 2Diabetes: Insulin-unabhängige renale SGLT-2Inhibition und neue DPP-4-Therapieoptionen", DDG-Jahrestagung, Stuttgart, Mai 2012 (Veranstalter: AstraZeneca und Bristol-Myers Squibb) 